Mohammad Roshan Soobratty ${ }^{1}$, Ruth Whitfield ${ }^{1}$, Krithika Subramaniam ${ }^{2}$, Grace Grove ${ }^{3}$, Anthony Carver ${ }^{3}$, Grace V. O'Donovan ${ }^{4}$, Houdini H.T. Wu ${ }^{5}$, Oona Y-C. Lee ${ }^{5}$, Ramasamyiyer Swaminathan ${ }^{2,3}$, Graham F. Cope ${ }^{5}$ and Heather J. Milburn ${ }^{3,4}$

${ }^{1}$ Dept of Respiratory Medicine, Mayday University Hospital, Croydon, ${ }^{2}$ Dept of Biochemistry, Guy's and St Thomas' NHS Foundation Trust, London, ${ }^{3}$ King's College London School of Medicine, University of London, London, ${ }^{4}$ Dept of Respiratory Medicine, Guy's and St Thomas' NHS Foundation Trust, London, and ${ }^{5}$ College of Medicine and Dentistry, University of Birmingham, Birmingham, UK.

Correspondence: H.J. Milburn, Chest Clinic, Guy’s Hospital, Great Maze Pond, London, SE1 9RT, UK.

E-mail: heather.milburn@gstt.nhs.uk

Received: July 312013 | Accepted after revision: Dec 062013 | First published online: Jan 162014

Support statement: This study was approved by Bexley and Greenwich Research Ethics Committee (London, UK) (Corec\#07/H0809/45). We thank the Sir Halley Stewart Trust for funding this study, which supported M.R. Soobratty.

Conflict of interest: Disclosures can be found alongside the online version of this article at www.erj.ersjournals.com

Acknowledgements: We are grateful to the TB patients, TB nurses and Respiratory Specialist Registrars at Mayday University Hospital (Croydon, UK) and Guy's and St Thomas' Hospitals (both London, UK) who contributed to the study.

\title{
References
}

Marais BJ, van Zyl S, Schaaf HS, et al. Adherence to isoniazid preventive chemotherapy: a prospective communitybased study. Arch Dis Child 2006; 91: 762-765.

2 Whitfield R, Cope GF. Point-of-care test to monitor adherence to anti-tuberculous treatment. Ann Clin Biochem 2004; 41: 411-413.

3 Schraufnagel DE, Stoner R, Whiting E, et al. Testing for isoniazid. An evaluation of the Arkansas method. Chest 1990; 98: 314-316.

4 Guerra RL, Conde MB, Efron A, et al. Point-of-care Arkansas method for measuring adherence to treatment with isoniazid. Respir Med 2010; 104: 754-757.

5 Subramanian K. Determination of acetylation status by genotyping and phenotyping. MSc Dissertation. University College London, London, UK, 2011.

6 Parkin DP, Vandenplas S, Botha FJH, et al. Trimodality of isoniazid elimination: phenotype and genotype in patients with tuberculosis. Am J Respir Crit Care Med 1997; 155: 1717-1722.

Doll MA, Hein DW. Comprehensive human NAT2 genotype method using single nucleotide polymorphismspecific polymerase chain reaction primers and fluorogenic probes. Anal Biochem 2001; 288: 106-108.

8 Nicolau I, Tian L, Menzies D, et al. Point-of-care urine tests for smoking status and isoniazid treatment monitoring in adult patients. PLoS One 2012; 7: e45913.

9 Eidus L, Glatthaar E, Hodgkin MM, et al. Comparison of isoniazid phenotyping of black and white patients with emphasis on South African blacks. Int J Clin Pharmacol Biopharm 1979; 17: 311-316.

10 Anitha A, Banerjee M. Arylamine $\mathrm{N}$-acetyltransferase 2 polymorphism in the ethnic populations of South India. Int J Mol Med 2003; 11: 125-131.

\section{Detecting active pulmonary tuberculosis with a breath test using nanomaterial- based sensors}

\section{To the Editor:}

Detecting active tuberculosis (TB) remains a major global public health challenge [1]. The tuberculin skin test does not distinguish latent from active TB [2]. The interferon- $\gamma$ release assays have similar limitations [3]. Acid-fast bacilli staining of sputum has a high false-negative rate (up to 50\%) [4]. Nucleic acid amplification tests (NAATs), such as GeneXpert MTB/RIF (Cepheid, Sunnyvale, CA, USA), are accurate but require a good infrastructure and the necessity to obtain a good quality sputum sample, which is often unobtainable in more than a third of HIV-infected persons $[5,6]$.

Given these unmet needs, we explored the use of a novel, rapid, simple and inexpensive point-of-care test for the diagnosis of TB [7]. The approach is based on the detection of volatile organic compounds (VOCs) that are emitted from infected cells and released in exhaled breath $[8,9]$. Using gas chromatography linked with mass spectrometry, researchers have previously reported identification of TB-related VOCs in the exhaled breath, though there has been low accuracy in detection (80-85\%) [10]. In this study, we explore 
the possibility of active TB detection via the analysis of exhaled breath using a novel technology of organically modified nanomaterial-based sensors. Such cross-reactive sensors are highly sensitive to the collective changes in the VOCs spectrum [8].

Therefore, we designed a case-control study, in which breath and sputum sampling was performed in 210 adult participants, after informed consent, at three sites in Cape Town, South Africa, between November 2011 and March 2012. The study population consisted of two main subgroups. The first subgroup included those in whom Mycobacterium tuberculosis culture was proven $(n=64)$. The control subgroup consisted of two main subcategories: 1$)$ healthy volunteers $(n=67)$; and 2$)$ a group of TB-negative subjects $(n=67)$. The latter comprised those in whom TB was suspected yet presented a negative smear microscopy, culture and GeneXpert MTB/RIF. The two groups were age and sex matched, yet differed in their smoking habits, HIV infection rates and treatment for TB.

Exhaled alveolar breath was collected in a controlled manner as reported elsewhere [11]. Briefly, inhaled air was cleared of ambient contaminants by repeatedly inhaling via a mouthpiece that contained a charcoal filter cartridge (Eco Medics, Dürnten, Switzerland), then, $750 \mathrm{~mL}$ of end tidal expired air was collected and trapped in two-bed ORBO 420 Tenax TA sorption tubes for gas sampling (Sigma-Aldrich, Shanghai, China). Sealed tubes were stored under refrigeration at $4^{\circ} \mathrm{C}$, until the day of analysis.

Thermal desorption was used to release the VOCs trapped in the tubes and delivered into a stainlesssteel test chamber containing 12 nanomaterial-based sensors (S1-S12), which are based on either: 1) molecularly-modified gold nanoparticles; or 2) molecularly-modified single-walled carbon nanotubes. More details on the fabrication and modification of these sensors can be found in $[12,13]$. The interaction between the films of the modified particles and the VOCs results in a time-dependent and reversible change in resistance, which was recorded and then normalised according to a daily calibration measurement of fixed standard.

Receiver operating characteristic (ROC) curves were applied to all sensors. The sensitivity, specificity, accuracy, positive predictive value (PPV) and negative predictive value (NPV) were calculated for both the training and validation sets. Statistical tests were performed using SAS JMP, version 10.0 (SAS Institute Inc., Marlow, UK).

Of the eligible 198 samples, 138 (44 TB positive, 47 TB negative and 47 healthy) were of known identity and were used as training set. The remaining independent 60 samples, of unknown identity (20 of each subgroup), were used for blind validation.

We used the ROC-derived Youden's index as a cut-point (best sensitivity + specificity - 1) to evaluate the examined sensors as a binary classifier. Three of the 12 sensors showed $>80 \%$ accuracy in classification. However, the best classifier was obtained from S1, a chemiresistor based on dodecanthiol-capped gold nanoparticles. S1 correctly classified 121 of the 138 training samples, an accuracy of $88 \%$; the sensitivity was $85 \%$ and the specificity $89 \%$ (fig. 1a). The ROC's area under curve (AUC) was $94.8 \%$ (fig. 1b). In the validation set, sensitivity was $90 \%$, specificity was $93 \%$ and accuracy was $92 \%$. Moreover, when applying a cut-off to rule out TB (a threshold scoring 95\% sensitivity in the training phase), the specificity, PPV and NPV were $83 \%, 78 \%$, and $96 \%$, respectively, in the training set. In the blinded validation set, the relevant values were $88 \%, 76 \%$ and $94 \%$, respectively. In the threshold to rule-in TB (a specificity of $96 \%$ in the training phase), the sensitivity, PPV and NPV were $68 \%, 93 \%$ and $87 \%$, respectively, in the training set. In the blind test, the relevant values were $78 \%, 76 \%$ and $94 \%$, respectively (fig. 1a).

The effects of potential confounding factors were examined. S1 showed a random classification ability between smokers versus non-smokers, HIV-positive versus HIV-negative subjects and treated versus untreated TB (ROC AUC of 56\%, 54\% and 55\%, respectively) (fig. 1c). This proved that the results were unaffected by confounding factors.

In the present study, we used NAAT as a complementary diagnostic test to verify a true-negative result in subjects who were found to be negative with a sputum test [6], and were known to have relatively low sensitivity, yet a very high $(\sim 100 \%)$ specificity [4].

One obvious advantage of breath analysis over sputum tests is the simplicity of obtaining the sample; this is in contrast to the difficulties in obtaining sputum both in children and HIV patients [5, 6]. The lack of confounding by HIV is another clear advantage over the existing TB diagnostic tests, which exhibit up to $43 \%$ misclassification in HIV-TB co-infected persons [14]. These results could be explained by the fact that nanomaterial-based sensors are broadly cross-reactive, so that each responds to a substantial number of VOCs of interest (e.g. 2,4-dimethylheptane, acetylaldhedye and 2-butanone [10]). Thus, the response is the result of the whole mixture of breath VOCs of interest, rather than a single VOC that may be linked with HIV $[8,15]$. 

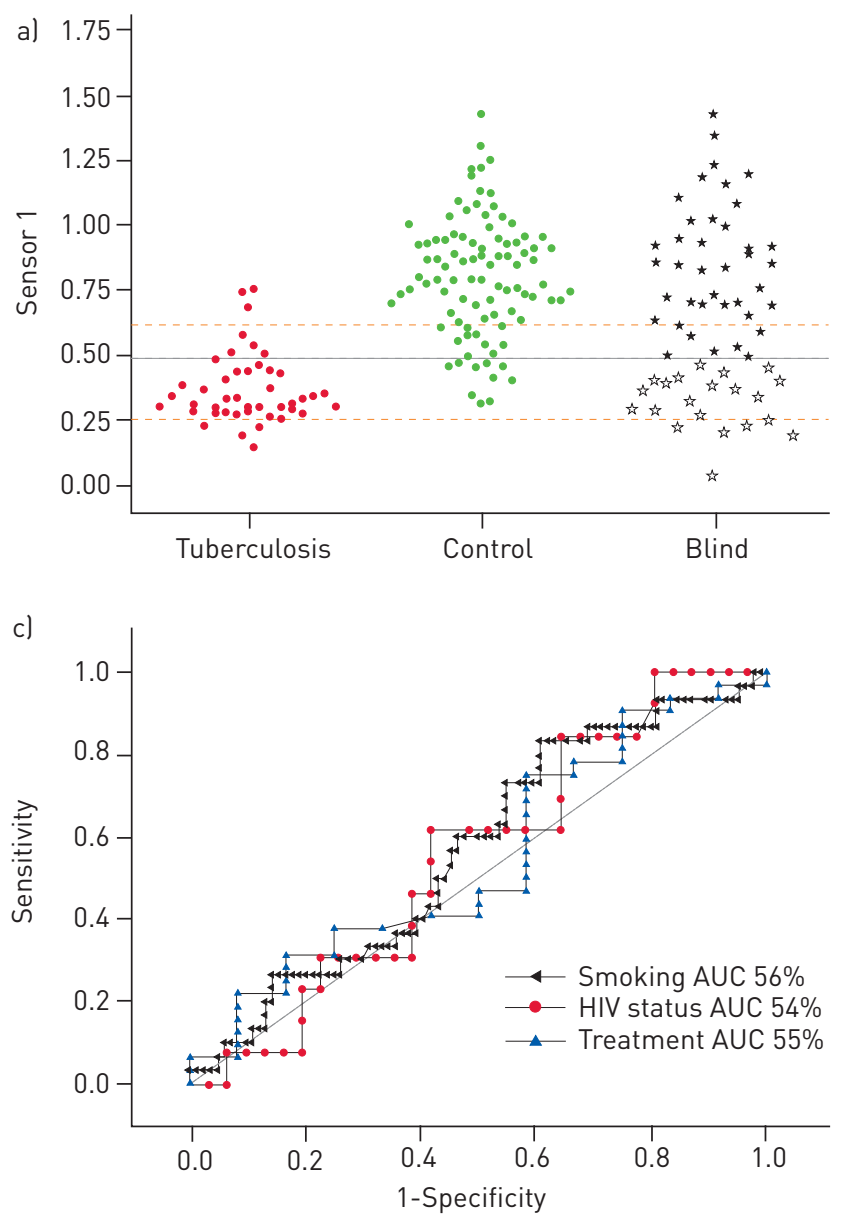

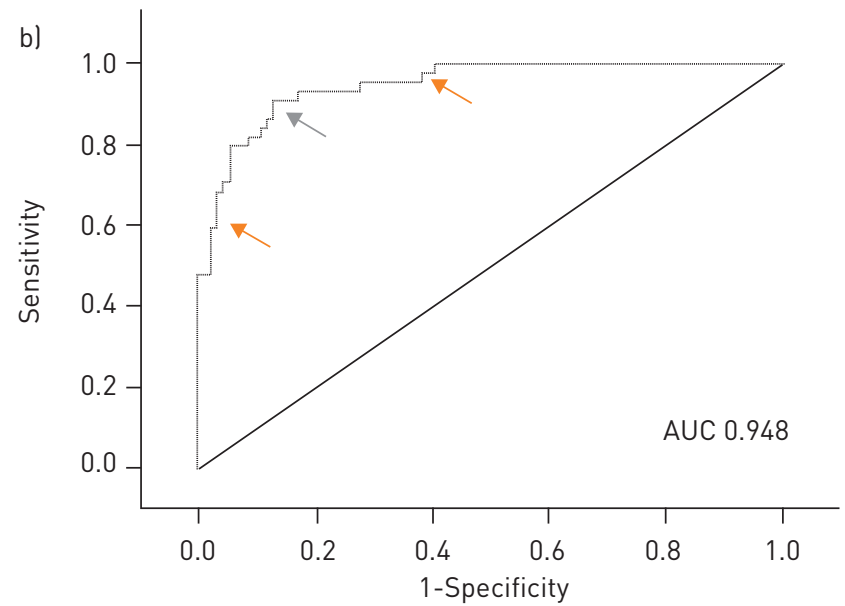

FIGURE 1 a) Dot-plots of the response of sensor S1. Each circle/star represents one sample. The central dashed line represents Youden's cut-point, and the outer dashed lines represents the cut-points to rule-in and rule-out tuberculosis. Samples from the validation set with responses lower than the threshold were classified as tuberculosis positive (open stars) or non-tuberculosis positive (closed stars) according to Youden's cut-point. b) Receiver operating characteristic (ROC) curve of sensor S1. C) ROC curves of sensor S1 when comparing smoking, HIV and treatment status. AUC: area under curve.

Our study has several limitations. The case-control design may over-estimate sensitivity and specificity; thus, our results must be interpreted with caution. We also didn't exclude latent TB cases. The presented results only apply to a high HIV-TB setting in Africa; further studies are therefore required in different settings. Due to safety regulations, healthy subjects were sampled in a separate location from subjects with suspected $\mathrm{TB}$, which could raise concerns regarding possible geographical bias affecting the results. We compared the healthy samples collected in one location with TB-negative samples collected in other locations. The results did not show any difference between the two control subgroups (AUC ROC 58\%), thus dismissing the fear of a potential geographical effect. Another limitation of the study is that many TB patients were treated prior to breath sampling (median of 5 days), which may have influenced our results. However, this was unavoidable for ethical reasons, and the analysis of the subgroups did not show any effect of TB treatment.

In summary, as $\mathrm{TB}$ diagnostics are usually either inaccurate or too expensive and complicated for use as a point-of-care detection tool, we examined a noninvasive, relatively simple and cheap (US\$1 per sensor) methodology to fill the gap. The preliminary proof-of-concept results indicate that a nanomaterial-based sensor may be a useful rule-in or rule-out tool for the diagnosis of TB ( $92 \%$ accuracy). A larger cohort study is required to validate the results. analysis http://ow.ly/tOPao

Morad K. Nakhleh ${ }^{1}$, Raneen Jeries ${ }^{1}$, A'laa Gharra $^{1}$, Anke Binder ${ }^{2}$, Yoav Y. Broza ${ }^{1}$, Mellissa Pascoe ${ }^{2}$, Keertan Dheda ${ }^{2}$ and Hossam Haick ${ }^{1}$

${ }^{1}$ Dept of Chemical Engineering and Russell Berrie Nanotechnology Institute, Technion - Israel Institute of Technology, Haifa, Israel. ${ }^{2}$ Division of Pulmonology and UCT Lung Institute, Dept of Medicine, Groote Schuur Hospital Observatory, Cape Town, South Africa. 
Correspondence: H. Haick, Dept of Chemical Engineering and Russell Berrie Nanotechnology Institute, Technion - Israel Institute of Technology, Haifa 3200003, Israel. E-mail: hhossam@tx.technion.ac.il

Received: Jan 292014 | Accepted after revision: Feb 142014

Conflict of interest: Disclosures can be found alongside the online version of this article at www.erj.ersjournals.com

Acknowledgments: The authors acknowledge Dr Falk Fish from Orgenics Ltd (Yavne, Israel). Orgenics Ltd have expressed interest in commercialising this technology and provided funding this study. The company played no role in the study design, analysis of the data, or drafting of the manuscript.

\title{
References
}

World Health Organization. Global Tuberculosis Control 2010. Geneva, World Health Organization, 2010.

2 American Thoracic Society/Centers for Disease Control and Prevention. Targeted tuberculin testing and treatment of latent tuberculosis infection. ATS/CDC Statement Committee on Latent Tuberculosis Infection membership list, June 2000. http://www.cdc.gov/MMWR/PREVIEW/MMWRHTML/rr4906a1.htm

3 Dheda K, van Zyl Smit R, Badri M, et al. T-cell interferon- $\gamma$ release assays for the rapid immunodiagnosis of tuberculosis: clinical utility in high-burden vs. low-burden settings. Curr Opin Pulm Med 2009; 15: 188-200.

$4 \quad$ Lawn SD, Zumla AI. Tuberculosis. Lancet 2011; 378: 57-72.

5 Whiley DM, Tapsall JW, Sloots TP. Nucleic acid amplification testing for Neisseria gonorrhoeae: an ongoing challenge. J Mol Diagn 2006; 8: 3-15.

6 Theron G, Peter J, Meldau R, et al. Accuracy and impact of Xpert MTB/RIF for the diagnosis of smear-negative or sputum-scarce tuberculosis using bronchoalveolar lavage fluid. Thorax 2013; 68: 1043-1051.

7 Wallis RS, Pai M, Menzies D, et al. Biomarkers and diagnostics for tuberculosis: progress, needs, and translation into practice. Lancet 2010; 375: 1920-1937.

8 Broza YY, Kremer R, Tisch U, et al. A nanomaterial-based breath test for short-term follow-up after lung tumor resection. Nanomedicine 2013; 9: 15-21.

9 Wheelock CE, Goss VM, Balgoma D, et al. Application of 'omics technologies to biomarker discovery in inflammatory lung diseases. Eur Respir J 2013; 42: 802-825.

10 Phillips M, Cataneo RN, Condos R, et al. Volatile biomarkers of pulmonary tuberculosis in the breath. Tuberculosis 2007; 87: 44-52.

11 Peled N, Hakim M, Bunn PA Jr, et al. Non-invasive breath analysis of pulmonary nodules. J Thorac Oncol 2012; 7: $1528-1533$.

12 Dovgolevsky E, Tisch U, Haick H. Chemically sensitive resistors based on monolayer-capped cubic nanoparticles: towards configurable nanoporous sensors. Small 2009; 5: 1158-1161.

13 Peng G, Tisch U, Adams O, et al. Diagnosing lung cancer in exhaled breath using gold nanoparticles. Nat Nanotechnol 2009; 4: 669-673.

14 Elliott A, Namaambo K, Allen B, et al. Negative sputum smear results in HIV-positive patients with pulmonary tuberculosis in Lusaka, Zambia. Tubercle and Lung Disease 1993; 73: 191-194.

15 Greulich T, Hattesohl A, Grabisch A, et al. Detection of obstructive sleep apnoea by an electronic nose. Eur Respir J 2013; 42: 145-155.

\section{Rituximab in the treatment of refractory pulmonary sarcoidosis}

\author{
To the Editor:
}

Sarcoidosis is a chronic disease characterised by granulomatous depositions that can occur in virtually any organ system [1]. Currently, there is no US Food and Drug Administration (FDA)-approved therapy for sarcoidosis; however, corticosteroids have proven efficacious and are a commonly used treatment [2]. In patients with chronic or pulmonary disease who do not respond to corticosteroids, or in whom steroid use is contraindicated, agents such as methotrexate, azathioprine and tumour necrosis factor (TNF)- $\alpha$ antagonists may be effective $[3,4]$. However, a need persists for patients who fail to respond to current options.

Sarcoidosis is a T-cell-mediated disease; however, humoral mechanisms may play a role in its pathogenesis [5]. Sarcoidosis is often associated with hypergammaglobulinaemia, autoantibody production and circulating immune complexes [6].

B-cell-targeted therapies have shown positive results in many T-cell-mediated autoimmune diseases. Rituximab is a chimeric monoclonal antibody that causes depletion of CD20 ${ }^{+} \mathrm{B}$-cells [7]. Rituximab is FDA approved for the treatment of rheumatoid arthritis, granulomatosis with polyangiitis (Wegener's) and microscopic polyangiitis, and is also being studied in Sjögren's syndrome, systemic lupus erythematosus 